\title{
Analytic investigation of the $(2+1)$-dimensional Schwarzian Korteweg-de Vries equation for traveling wave solutions
}

\author{
İsmail Aslan \\ Department of Mathematics, Izmir Institute of Technology, Urla, Izmir 35430, Turkey
}

\section{A R T I C L E I N F O}

\section{Keywords:}

Exp-function method

Extended $\left(\mathrm{G}^{\prime} / \mathrm{G}\right)$-expansion method

Schwarzian Korteweg-de Vries equation

Traveling wave solutions

Solitary waves

\begin{abstract}
A B S T R A C T
By means of the two distinct methods, the Exp-function method and the extended $\left(\mathrm{G}^{\prime} / \mathrm{G}\right)$ expansion method, we successfully performed an analytic study on the $(2+1)$-dimensional Schwarzian Korteweg-de Vries equation. We exhibited its further closed form traveling wave solutions which reduce to solitary and periodic waves. New rational solutions are also revealed.
\end{abstract}

(c) 2011 Elsevier Inc. All rights reserved.

\section{Introduction}

The Korteweg-de Vries (KdV) equation [1] has been a milestone in the study of nonlinear science since it possess a wealth of interesting and crucial properties. The fact that many integrable equations [2] are originated from the KdV equation is well known. The most fundamental one, within the class of KdV related equations, is the Schwarzian Korteweg-de Vries equation $(\mathrm{SKdV})[3]$

$$
\frac{\phi_{t}}{\phi_{x}}+\left(\frac{\phi_{x x}}{\phi_{x}}\right)_{x}-\frac{1}{2}\left(\frac{\phi_{x x}}{\phi_{x}}\right)^{2}=0
$$

Several generalizations and extensions of Eq. (1) appeared in the literature. One model, in the context of integrable (2+1)dimensional equations, is

$$
W_{t}+\frac{1}{4} W_{x x z}-\frac{W_{x} W_{x z}}{2 W}-\frac{W_{x x} W_{z}}{4 W}+\frac{W_{x}^{2} W_{z}}{2 W^{2}}-\frac{W_{x}}{8}\left(\partial_{x}^{-1}\left(\frac{W_{x}^{2}}{W^{2}}\right)\right)_{z}=0
$$

where $\partial_{x}^{-1} f=\int f d x$. Eq. (2) is called $(2+1)$-dimensional Schwarzian Korteweg-de Vries $((2+1)$-D SKdV) equation. Toda and $\mathrm{Yu}$ [4] proved the integrability of (2) in the sense of the Weiss-Tabor-Carnevale Painlevé expansion. By means of the transformations $W=\phi_{x}, \phi=\exp (\eta), u=\eta_{x}, v=\eta_{t}$, Eq. (2) can be converted into a coupled nonlinear system in local form as

$$
4 u^{2} v_{x}-4 u u_{x} v+u^{2} u_{x x z}-u u_{x x} u_{z}-3 u u_{x} u_{x z}+3 u_{x}^{2} u_{z}-u^{4} u_{z}=0, u_{t}-v_{x}=0
$$

It is remarkable that the system (3) is related to the Ablowitz-Kaup-Newell-Segur (AKNS) equation via a Miura transformation [5]. As a result, being of great interest in both mathematics and physics, a great deal of research work has been invested for SKdV type equations [6-13].

Our objective in this study, by using two of the most recent expansion methods, is to perform an analytic study on the system (3) in order to derive as widest families of solutions as possible. Our preference is the Exp-function method [14] and an extended version of the $\left(G^{\prime} / G\right)$-expansion method [15]. Both methods have the capabilities of providing not only more

E-mail address: ismailaslan@iyte.edu.tr 
general forms of solutions but also periodic and solitary waves. The rest of this paper is organized as follows. In Section 2 , we describe the Exp-function and the extended $\left(G^{\prime} / G\right)$-expansion methods for solving NEEs. In Section 3, we illustrate our procedures in detail with the system (3). Finally, a conclusion is given in Section 4.

\section{Methodology}

In this section, we briefly highlight the main features of the Exp-function and the extended $\left(\mathrm{G}^{\prime} / \mathrm{G}\right)$-expansion methods [16-28]. Let us consider a PDE for $u(x, z, t)$ in the form

$$
P\left(u, u_{t}, u_{x}, u_{z}, u_{t t}, u_{t x}, u_{t z}, u_{x x}, u_{x z}, u_{z z}, \ldots\right)=0,
$$

where $P$ is a polynomial in its arguments. By the transformation $u(x, z, t)=U(\xi), \xi=k x+m z+n t$, where $k$, $m$ and $n$ are nonzero arbitrary constants, Eq. (4) can be reduced into an ODE of the form

$$
Q\left(U, U^{\prime}, U^{\prime \prime}, \ldots\right)=0
$$

where primes denote derivatives with respect to $\xi$. We consider two distinct approaches to Eq. (5):

\subsection{The Exp-function method}

We assume that Eq. (5) admits traveling wave solutions of the form

$$
U(\xi)=\frac{a_{c} \exp (c \xi)+\cdots+a_{-d} \exp (-d \xi)}{b_{p} \exp (p \xi)+\cdots+b_{-q} \exp (-q \xi)},
$$

where $c, d, p$ and $q$ are unknown positive integers determined by the homogeneous balance principle, $a_{i}$ and $b_{j}$ are unknown constants. Substituting (6) into Eq. (5) leads to a system of nonlinear algebraic equations for $a_{i}, b_{j}, k, m$ and $n$. Finally, substitution of the system's solutions into (6) gives traveling wave solutions to Eq. (4).

\subsection{The extended $\left(G^{\prime} / G\right)$-expansion method}

We initially predict the structure of the solution $U=U(\xi)$ to Eq. (5) in the finite series form

$$
U=\sum_{i=-n}^{n} a_{i}\left(\frac{G^{\prime}}{G}\right)^{i}, \quad G^{\prime \prime}+\lambda G^{\prime}+\mu G=0
$$

where $G=G(\xi)$ and primes denote derivatives with respect to $\xi ; a_{i}, \lambda$ and $\mu$ are constants to be specified later. The positive integer $n$ can be determined by the homogeneous balance method. Substituting (7) into Eq. (5) yields a system of nonlinear algebraic equations for $a_{i}, \lambda, \mu, k, m$ and $n$. Finally, substitution of the system's solutions into (7) gives traveling wave solutions to Eq. (4).

\section{Analysis}

By means of the wave transformation $u(x, z, t)=U(\xi), v(x, z, t)=V(\xi), \xi=k x+m z+n t$, where $k, m$ and $n$ are constants, the system (3) turns into

$$
3 k^{2} m\left(U^{\prime}\right)^{3}-m U^{4} U^{\prime}-4 k^{2} m U U^{\prime} U^{\prime \prime}+k^{2} m U^{2} U^{\prime \prime \prime}-4 k U U^{\prime} V+4 k U^{2} V^{\prime}=0, \quad n U^{\prime}-k V^{\prime}=0,
$$

where primes denote derivatives with respect to $\xi$. Integrating the second equation of (8) once and solving the resulting expression for $V$, we get $V=(n / k) U+C$, where $C$ is an integration constant. Hence, the first equation of (8) becomes

$$
3 k^{2} m\left(U^{\prime}\right)^{3}-m U^{4} U^{\prime}-4 k^{2} m U U^{\prime} U^{\prime \prime}+k^{2} m U^{2} U^{\prime \prime \prime}-4 k C U U^{\prime}=0 .
$$

\subsection{Using the Exp-function method}

We consider the ansatz (6) for the solution of Eq. (9). Balancing the terms $\left(U^{\prime}\right)^{3}$ and $U^{4} U^{\prime}$ leads to $p=c$ and $q=d$. Now, we consider the case $p=c=1$ and $d=q=1$. Thus, the solution of Eq. (9) can be expressed as

$$
U(\xi)=\frac{a_{1} \exp (\xi)+a_{0}+a_{-1} \exp (-\xi)}{b_{1} \exp (\xi)+b_{0}+b_{-1} \exp (-\xi)} .
$$

Substituting (10) into Eq. (9), we get the generalized solitary wave solutions to the system (3):

$$
u_{1}^{\mp}(x, z, t)=\frac{k^{2} \exp (k x+m z+n t)}{-a_{0} \mp k\left(b_{0}+\exp (k x+m z+n t)\right)}, \quad v_{1}^{\mp}(x, z, t)=\frac{k n \exp (k x+m z+n t)}{-a_{0} \mp k\left(b_{0}+\exp (k x+m z+n t)\right)} ;
$$




$$
\begin{aligned}
& u_{2}(x, z, t)=\frac{4 k^{2} a_{0} \exp (k x+m z+n t)}{-a_{0}^{2}+k^{2}\left(b_{0}+2 \exp (k x+m z+n t)\right)^{2}}, \quad v_{2}(x, z, t)=\frac{4 k n a_{0} \exp (k x+m z+n t)}{-a_{0}^{2}+k^{2}\left(b_{0}+2 \exp (k x+m z+n t)\right)^{2}} ; \\
& u_{3}^{\mp}(x, z, t)=\frac{2 k a_{-1}}{\mp a_{-1}+2 k \exp (2(k x+m z+n t))}, \quad v_{3}^{\mp}(x, z, t)=\frac{2 n a_{-1}}{\mp a_{-1}+2 k \exp (2(k x+m z+n t))} \\
& u_{4}^{\mp}(x, z, t)=\frac{k a_{0}}{\mp a_{0}+k \exp (k x+m z+n t)}, \quad v_{4}^{\mp}(x, z, t)=\frac{n a_{0}}{\mp a_{0}+k \exp (k x+m z+n t)},
\end{aligned}
$$

where $a_{0}, b_{0}, k, m, n$ are arbitrary constants. Here and henceforth, the signs $( \pm)$ or ( $\mp$ ) are ordered vertically.

Remark 1. By setting special values to the arbitrary parameters, we can construct formal solitary and periodic wave solutions to (3). As a special example, if we let $a_{0}=\left(\mp 1-b_{0}\right) k$ in the $(-)$ branch of (11) then we get formal solitary waves to (3) as

$$
\begin{array}{ll}
u_{5}(x, z, t)=-\frac{k}{2}\left(1+\tanh \frac{1}{2}(k x+m z+n t)\right), & v_{5}(x, z, t)=-\frac{n}{2}\left(1+\tanh \frac{1}{2}(k x+m z+n t)\right) \\
u_{6}(x, z, t)=-\frac{k}{2}\left(1+\operatorname{coth} \frac{1}{2}(k x+m z+n t)\right), & v_{6}(x, z, t)=-\frac{n}{2}\left(1+\operatorname{coth} \frac{1}{2}(k x+m z+n t)\right)
\end{array}
$$

where $k, m, n$ are arbitrary constants.

Moreover, if $k, m, n$ are imaginary numbers in the complex variation $\xi=k x+m z+n t$, say $k=i K, m=i M, n=i N, i^{2}=-1$, then using the transformation

$$
\exp ( \pm \xi)=\exp ( \pm i(K x+M z+N t))=\cos (K x+M z+N t) \pm i \sin (K x+M z+N t)
$$

we can obtain periodic solutions. For example, taking (17) into consideration, (12) becomes

$$
\begin{aligned}
& u_{7}(x, z, t)=\frac{4 K^{2} a_{0}}{\left(\left(2 i K-a_{0}-i K b_{0}\right) \sin \left(\frac{K x+M z+N t}{2}\right)+\left(2 K-i a_{0}+K b_{0}\right) \cos \left(\frac{K x+M z+N t}{2}\right)\right)\left(\left(2 i K+a_{0}-i K b_{0}\right) \sin \left(\frac{K x+M z+N t}{2}\right)+\left(2 K+i a_{0}+K b_{0}\right) \cos \left(\frac{K x+M z+N t}{2}\right)\right)}, \\
& v_{7}(x, z, t)=\frac{4 K N a_{0}}{\left(\left(2 i K-a_{0}-i K b_{0}\right) \sin \left(\frac{K x+M z+N t}{2}\right)+\left(2 K-i a_{0}+K b_{0}\right) \cos \left(\frac{K x+M z+N t}{2}\right)\right)\left(\left(2 i K+a_{0}-i K b_{0}\right) \sin \left(\frac{K x+M z+N t}{2}\right)+\left(2 K+i a_{0}+K b_{0}\right) \cos \left(\frac{K x+M z+N t}{2}\right)\right)} .
\end{aligned}
$$

Now, letting " $b_{0}=0, a_{0}=\mp 2 K$ " or " $b_{0}=0, a_{0}=\mp 2 i K$ ” in (18) gives periodic solutions to ( 3 ) as

$$
\begin{array}{ll}
u_{8}^{\mp}(x, z, t)=\mp \frac{K}{\cos (K x+M z+N t)}, & v_{8}^{\mp}(x, z, t)=\mp \frac{N}{\cos (K x+M z+N t)} ; \\
u_{9}^{\mp}(x, z, t)=\mp \frac{K}{\sin (K x+M z+N t)}, & v_{9}^{\mp}(x, z, t)=\mp \frac{N}{\sin (K x+M z+N t)},
\end{array}
$$

where $K, M$ and $N$ are arbitrary constants.

\subsection{Using the extended $\left(G^{\prime} / G\right)$-expansion method}

Now, we assume that the solution of Eq. (9) can be expressed as the ansatz (7). By the homogeneous balance principle, we determine that $n=1$. Hence, we look for solutions to Eq. (9) in the form

$$
U=a_{0}+a_{1}\left(\frac{G^{\prime}}{G}\right)+a_{-1}\left(\frac{G^{\prime}}{G}\right)^{-1}, \quad G^{\prime \prime}+\lambda G^{\prime}+\mu G=0 .
$$

Substituting (21) into Eq. (9), we get the traveling wave solutions to the system (3):

$$
\begin{aligned}
& u_{10}^{\mp}(x, z, t)=\mp k\left(w(x, z, t)+\frac{\mu}{w(x, z, t)}\right), \quad v_{10}^{\mp}(x, z, t)=\mp n\left(w(x, z, t)+\frac{\mu}{w(x, z, t)}\right) ; \\
& u_{11}^{\mp}(x, z, t)=-k(w(x, z, t) \pm i \sqrt{\mu}), \quad v_{11}^{\mp}(x, z, t)=-n(w(x, z, t) \pm i \sqrt{\mu}) ; \\
& u_{12}^{\mp}(x, z, t)=k(w(x, z, t) \mp i \sqrt{\mu}), \quad v_{12}^{\mp}(x, z, t)=n(w(x, z, t) \mp i \sqrt{\mu}) ; \\
& u_{13}^{\mp}(x, z, t)=k\left(w(x, z, t)-\frac{\mu}{w(x, z, t)}\right) \mp 2 i k \sqrt{\mu}, \quad v_{13}^{\mp}(x, z, t)=n\left(w(x, z, t)-\frac{\mu}{w(x, z, t)}\right) \mp 2 i k \sqrt{\mu} \text {; } \\
& u_{14}^{\mp}(x, z, t)=-k\left(w(x, z, t)-\frac{\mu}{w(x, z, t)}\right) \mp 2 i k \sqrt{\mu}, \quad v_{14}^{\mp}(x, z, t)=-n\left(w(x, z, t)-\frac{\mu}{w(x, z, t)}\right) \mp 2 i k \sqrt{\mu} ; \\
& u_{15}^{\mp}(x, z, t)=k\left(\mp i \sqrt{\mu}-\frac{\mu}{w(x, z, t)}\right), \quad v_{15}^{\mp}(x, z, t)=n\left(\mp i \sqrt{\mu}-\frac{\mu}{w(x, z, t)}\right) ; \\
& u_{16}^{\mp}(x, z, t)=k\left(\mp i \sqrt{\mu}+\frac{\mu}{w(x, z, t)}\right), \quad v_{16}^{\mp}(x, z, t)=n\left(\mp i \sqrt{\mu}+\frac{\mu}{w(x, z, t)}\right) ;
\end{aligned}
$$




$$
\begin{aligned}
& u_{17}^{\mp}(x, z, t)=\mp k\left(w(x, z, t)+\frac{\mu}{w(x, z, t)}\right)+2 k \sqrt{\mu}, \quad v_{17}^{\mp}(x, z, t)=\mp n\left(w(x, z, t)+\frac{\mu}{w(x, z, t)}\right)+2 n \sqrt{\mu}-4 k^{2} m \mu^{3 / 2} \\
& u_{18}^{\mp}(x, z, t)=\mp k\left(w(x, z, t)+\frac{\mu}{w(x, z, t)}\right)-2 k \sqrt{\mu}, \quad v_{18}^{\mp}(x, z, t)=\mp n\left(w(x, z, t)+\frac{\mu}{w(x, z, t)}\right)-2 n \sqrt{\mu}+4 k^{2} m \mu^{3 / 2},
\end{aligned}
$$

where the function $w(x, z, t)$ is defined as

$$
\begin{aligned}
& w(x, z, t)=\sqrt{-\mu}\left[\frac{C_{1} \cosh \sqrt{-\mu}(k x+m z+n t)+C_{2} \sinh \sqrt{-\mu}(k x+m z+n t)}{C_{1} \sinh \sqrt{-\mu}(k x+m z+n t)+C_{2} \cosh \sqrt{-\mu}(k x+m z+n t)}\right], \quad \mu<0, \\
& w(x, z, t)=\sqrt{\mu}\left[\frac{C_{2} \cos \sqrt{\mu}(k x+m z+n t)-C_{1} \sin \sqrt{\mu}(k x+m z+n t)}{C_{1} \cos \sqrt{\mu}(k x+m z+n t)+C_{2} \sin \sqrt{\mu}(k x+m z+n t)}\right], \quad \mu>0, \\
& w(x, z, t)=\frac{C_{1}}{C_{1}(k x+m z+n t)+C_{2}}, \quad \mu=0
\end{aligned}
$$

in which $C_{1}, C_{2}, k, m, n$ and $\mu$ are arbitrary constants.

Remark 2. By substituting (31)-(33) into the solution functions (22)-(30), we can construct three types of solutions to the system (3); hyperbolic, trigonometric, and rational. Moreover, assigning special values to the arbitrary parameters $C_{1}$ and $C_{2}$ gives solitary and periodic wave solutions. For instance, if one takes $C_{2} \neq 0$ and $C_{1}^{2}<C_{2}^{2}$ in (31) and (32) then the function $w(x, z, t)$ becomes

$$
\begin{aligned}
& w(x, z, t)=\sqrt{-\mu} \tanh \left(\sqrt{-\mu}(k x+m z+n t)+\tanh ^{-1}\left(C_{1} / C_{2}\right)\right), \quad \mu<0, \\
& w(x, z, t)=\sqrt{\mu} \cot \left(\sqrt{\mu}(k x+m z+n t)+\tan ^{-1}\left(C_{1} / C_{2}\right)\right), \quad \mu>0,
\end{aligned}
$$

so that (34) and (35) provides the desired solitary and periodic wave solutions to (3).

Remark 3. It is obvious that our results are wider than the ones in [29] and the rational solutions provided by (33) do not appear in there.

Remark 4. Any of the existing techniques for solving NEEs can have some advantages and disadvantages. The Exp-function and the extended $\left(\mathrm{G}^{\prime} / \mathrm{G}\right)$-expansion methods are direct methods that usually give rise to families of solutions. However, nonlinear algebraic equations that we may need to perform to carry the techniques to completion may, for some nonlinear problems, be rather difficult. There is no a general formula for solutions of nonlinear equations. Although a nonlinear equation may well have a solution involving an arbitrary parameter, there may also be other solutions. We attempted to derive as many solutions to the system (3) as possible by our methods. The obtained solutions might make good physical sense in applications.

\section{Conclusion}

Using the Exp-function and the extended $\left(\mathrm{G}^{\prime} / \mathrm{G}\right)$-expansion methods, we reported further exact traveling wave solutions to the $(2+1)$-D SKdV equation which admits physical significance in applications. Solitary and periodic waves are observed. In fact, our methods are entirely algorithmic and involve a large amount of tedious calculations which can become virtually unmanageable if attempted manually. However, the procedures do not require a large amount of CPU time with the aid of a computer algebra system. We assured the correctness of the obtained solutions by putting them back into the original equation with the aid of MATHEMATICA, it provides an extra measure of confidence in the results. Hence, the power of the employed methods is confirmed.

\section{References}

[1] E.M. de Jager, On the origin of the Korteweg-de Vries equation, 2006, arXiv:math/0602661v1.

[2] M.J. Ablowitz, P.A. Clarkson, Solitons, Nonlinear Evolution Equations and Inverse Scattering, Cambridge University Press, Cambridge, 1991.

[3] J. Weiss, The Painlevé property for partial differential equation. II: Bäcklund transformation, Lax pairs, and the Schwarzian derivative, J. Math. Phys. 24 (1983) 1405-1413.

[4] K. Toda, S. Yu, The investigation into the Schwarz-Korteweg-de Vries equation and the Schwarz derivative in (2+1), J. Math. Phys. 41 (2000) $4747-$ 4751.

[5] J. Ramirez, J.L. Romero, M.S. Bruzon, M.L. Gandarias, Multiple solutions for the Schwarzian Korteweg-de Vries equation in $(2+1)$ dimensions, Chaos Soliton. Fract. 32 (2007) 682-693.

[6] F. Guil, M. Manas, Loop algebras and the Krichever-Novikov equation, Phys. Lett. A 153 (1991) 90-94.

[7] G. Wilson, On the quasi-hamiltonian formalism of the KdV equation, Phys. Lett. A 132 (1988) 445-450.

[8] I. Ya Dorfman, The Krichever-Novikov equation and local symplectic structures, Sov. Math. Dolk. 38 (1989) 340-343.

[9] J. Ramirez, M.S. Bruzon, C. Muriel, M.L. Gandarias, The Schwarzian Korteweg-de Vries equation in (2+1) dimensions, J. Phys. A: Math. Gen. 36 (2003) $1467-1484$. 
[10] M.L. Gandarias, M.S. Bruzón, New solutions of the Schwarzian Korteweg-de Vries equation in $(2+1)$ dimensions based on weak symmetries, Theor. Math. Phys. 151 (2007) 752-761.

[11] J. Ramírez, J.L. Romero, New classes of solutions for the Schwarzian Korteweg-de Vries equation in $(2+1)$ dimensions, J. Phys. A: Math. Theor. 40 (2007) 4351-4365.

[12] D. Levi, M. Petrera, C. Scimiterna, The lattice Schwarzian KdV equation and its symmetries, J. Phys. A: Math. Theor. 40 (2007) 12753-12761.

[13] F. Calogero, M. Mariani, A modified Schwarzian Korteweg-de Vries Equation in $2+1$ dimensions with lots of isochronous solutions, Phys. Atom. Nuc. 68 (2005) 1646-1653.

[14] J.H. He, X.H. Wu, Exp-function method for nonlinear wave equations, Chaos Soliton. Fract. 30 (2006) 700-708.

[15] M. Wang, X. Li, J. Zhang, The ( $\left.G^{\prime} / G\right)$-expansion method and traveling wave solutions of nonlinear evolution equations in mathematical physics, Phys. Lett. A. $372(2008) 417-423$.

[16] T. Öziş, I. Aslan, Exact and explicit solutions to the (3+1)-dimensional Jimbo-Miwa equation via the Exp-function method, Phys. Lett. A 372 (2008) 7011-7015.

[17] İ. Aslan, Generalized solitary and periodic wave solutions to a (2+1)-dimensional Zakharov-Kuznetsov equation, Appl. Math. Comput. 217 (2010) $1421-1429$.

[18] i. Aslan, The Exp-function approach to the Schwarzian Korteweg-de Vries equation, Comput. Math. Appl. 59 (2010) $2896-2900$

[19] İ. Aslan, T. Özis, Analytic study on two nonlinear evolution equations by using the (G'/G)-expansion method, Appl. Math. Comput. 209 (2009) 425-429.

[20] İ. Aslan, T. Öziş, On the validity and reliability of the $\left(\mathrm{G}^{\prime} / \mathrm{G}\right)$-expansion method by using higher-order nonlinear equations, Appl. Math. Comput. 211 (2009) $531-536$.

[21] T. Öziş, İ. Aslan, Symbolic computation and construction of new exact traveling wave solutions to Fitzhugh-Nagumo and Klein-Gordon equations, Z. Naturforsch. 64a (2009) 15-20.

[22] T. Öziş, İ. Aslan, Symbolic computations and exact and explicit solutions of some nonlinear evolution equations in mathematical physics, Commun. Theor. Phys. 51 (2009) 577-580.

[23] İ. Aslan, Exact and explicit solutions to some nonlinear evolution equations by utilizing the $\left(\mathrm{G}^{\prime} / \mathrm{G}\right)$-expansion method, Appl. Math. Comput. 215 (2009) 857-863.

[24] T. Öziş, İ. Aslan, Application of the G'/G-expansion method to Kawahara type equations using symbolic computation, Appl. Math. Comput. 216 (2010) 2360-2365.

[25] i. Aslan, Discrete exact solutions to some nonlinear differential-difference equations via the (G'/G)-expansion method, Appl. Math. Comput. 215 (2009) 3140-3147.

[26] i. Aslan, The Ablowitz-Ladik lattice system by means of the extended (G'G)-expansion method, Appl. Math. Comput. 216 (2010) $2778-2782$.

[27] S. Zhang, W. Wang, J. Tong, A generalized $\left(\mathrm{G}^{\prime} / \mathrm{G}\right)$-expansion method and its application to the $(2+1)$-dimensional Broer-Kaup equations, Appl. Math. Comput. 209 (2009) 399-404.

[28] E.M.E. Zayed, K.A. Gepreel, Some applications of the ( $\left.\mathrm{G}^{\prime} / \mathrm{G}\right)$-expansion method to non-linear partial differential equations, Appl. Math. Comput. 212 (2009) $1-13$.

[29] T. Özer, New traveling wave solutions to AKNS and SKdV equations, Chaos Soliton. Fract. 42 (2009) 577-583. 